\title{
The Design and Implementation of User-defined Multi-table Query Algorithm
}

\author{
Zhenfang Shan ${ }^{1, a}$ and Hao $\mathrm{Kou}^{2, \mathrm{~b}}$ \\ ${ }^{1}$ Shijiazhuang Posts and Telecommunications Technical College, Hebei, China \\ ${ }^{2}$ Shijiazhuang Posts and Telecommunications Technical College, Hebei, China \\ azhenfangshan@163.com, bouhao@cptc.cn
}

Keywords: user-defined query; data dictionary; DWR

\begin{abstract}
Nowadays customers demand the query more and more flexible, they hope it is not restricted to the fixed query conditions, but through user's definition to configure query conditions for completing query operation. In a word, the query operation must be flexible enough. Base on this demand, we proposed user-defined multi-table query algorithm, and combined with data dictionary and DWR technology to achieve a practical financial network project. Since the success of the project on-line, we verified the feasibility and effectiveness of the algorithm.
\end{abstract}

\section{Introduction}

In recent years, the informatization trend of Chinese enterprises was in the ascendant. Enterprises have established their own information management system, built their own web server. And each database of the enterprise also has stored a lot of information. In these information systems, it inevitably involved data query and display as needed in each module. In the current query mode, generally multi-field fixed query in a table is very common, or there is a lot of input boxes with options in the front page. The back database query is also related to the interconnected multi-table query[1], but the condition of choices is still relatively fixed, the output display is also fixed, so users often complain that the query is not flexible enough. Users often need to define their own query conditions according to their need, and also the output display items can freely adjust the display order. In this paper, combined with the financial management information system, using user-defined query module as an example, focusing on the user-defined query design in front page for the interconnected multi-table of WEB management information system, we put forward our own solution.

\section{The description of user-defined query algorithm}

In the user-defined query, the difficult is to determine the arithmetic objects and arithmetic operators that users need to define, inside the operand is the field name or serial number, operators mainly contain logical and comparison operators. Users require complicated filtering conditions in order to meet business need, however, the condition will continue to change because of specific business or different situations, so how to find solutions in constant changes, this is the problem to be solved in the user-defined algorithm.

Suppose you want to do the conditional query for any relation table $\mathrm{R}$ in the information system database, it can be described by the following formula:

$$
\sigma_{\mathrm{F}}(\mathrm{R}) \equiv\{\mathrm{t} \mid \mathrm{t} \in \mathrm{R} \wedge \mathrm{F}(\mathrm{t})=\text { true }\}
$$

In it $\sigma$ is the choice operator, $F$ is the user-defined query conditional expression, $t$ is the tuple in relation table $\mathrm{R}, \sigma \mathrm{F}(\mathrm{R})$ represents the real tuple to meet the formula F selected from the relation table $\mathrm{R}$, they constitute a new relation table.

Conditional expression F of user-defined query usually consists of two parts, that is arithmetic symbols and operands, and their values in the algorithm are as follows: 
Operator: including the logical operators $L$ set and comparison operators $\theta$ set. The algorithm only takes $\wedge, \vee$ in the set $\mathrm{L}$ and $\%,<, \leqslant,>, \geqslant,=, \neq$ in the set $\theta . \%$ is containing elements.

Operands: Including tuple component (field name) or constant (column number).

For any relation table $\mathrm{R}$ which contains $\mathrm{K}$ elemental components, due to the combination and diversity and complexity required in the user-defined query, it brings complexity and uncertainty for F-functions. In order to meet user-defined query and user-defined relation table query, in software development and design process, we use the visualization window to show the structure of function $\mathrm{F}$, selection mode is given to the user, and constructed in accordance with the user-defined needs. If the user-defined constructed function $\mathrm{F}$ defined as $\mathrm{F}_{Z}$, the design of user-defined query can be expressed as constructing user conditional function $\mathrm{F}_{\mathrm{Z}}$ :

$$
\begin{aligned}
& \mathrm{F}_{\mathrm{Z}}=\left(\mathrm{M}_{1} \theta_{1} \mathrm{~V}_{1}\right) \mathrm{L}_{1}\left(\mathrm{M}_{2} \theta_{2} \mathrm{~V}_{2}\right) \ldots \mathrm{L}_{\mathrm{i}-1}\left(\mathrm{M}_{\mathrm{i}} \theta_{\mathrm{i}} \mathrm{V}_{\mathrm{i}}\right) \mathrm{L}_{\mathrm{i}} \ldots \\
& \sigma \mathrm{F}_{\mathrm{Z}}(\mathrm{R}) \equiv\left\{\mathrm{t} \mid \mathrm{t} \in \mathrm{R} \wedge \mathrm{F}_{\mathrm{Z}}(\mathrm{t})=\text { true }\right\}
\end{aligned}
$$

In it $\mathrm{M}_{\mathrm{i}}$ represents user-defined query components, $\theta_{\mathrm{i}}$ is the relational operators freely chosen by user, $V_{i}$ represents the corresponding range of user-defined query component, $L_{i}$ is the logic operators freely chosen by user. To meet this requirement, user can constructs user-defined query function $\mathrm{F}_{\mathrm{Z}}$ according to the following steps:

(1) Select the user-defined query components $\mathrm{M}_{\mathrm{i}}$;

(2) Select the relational operator $\theta_{i}$;

(3) Enter the user-defined query range $\mathrm{V}_{\mathrm{i}}$;

(4) If you continue to construct user-defined query conditions, select the logical operator $\mathrm{L}_{\mathrm{i}}$, then return to (1), (2), (3) continue to construct it, otherwise construction complete.

(5) Use relational expression $\sigma \mathrm{F}_{Z}(\mathrm{R}) \equiv\left\{\mathrm{t} \mid \mathrm{t} \in \mathrm{R} \wedge \mathrm{F}_{\mathrm{Z}}(\mathrm{t})=\right.$ true $\}$ to do user-defined query.

\section{The design of user-defined query algorithm}

We use an example to show the above process, the example is from our completed financial network project, the user-defined query of multi-related table and displayed object include "network query condition" and " information displayed for choosing"; Constructing combinational conditional table of user conditional function $\mathrm{F}_{\mathrm{Z}}$ (including the "table", "field", "conditions", "input values" four options); Constructing three buttons of user function F for controlling("+ (new)", "- (delete)", "query"). "select the information to display" window is used for users to select display items and display order, including table name, field names, operation, display field, display order five options, as shown in Figure 1. 


\begin{tabular}{|c|c|c|c|c|c|c|c|}
\hline \multicolumn{2}{|c|}{ 表 名( ‘table”) } & \multicolumn{2}{|c|}{ 字段名("fi=1d") } & \multicolumn{4}{|c|}{ 条件 ("conditions") 铪 入 值 ("input values") 操作 } \\
\hline 网点关键信息表 & $\checkmark$ & 所在行政区域 $\vee$ & & $=V$ & 福建省 & & + \\
\hline 业务收入信息表 & $\checkmark$ & 月份 & $\checkmark$ & $=\quad \vee$ & 2010-06 & - & + \\
\hline 业务收入信息表 & $\checkmark$ & 储葘收入 & $\checkmark$ & $>V$ & 10 & - & + \\
\hline
\end{tabular}

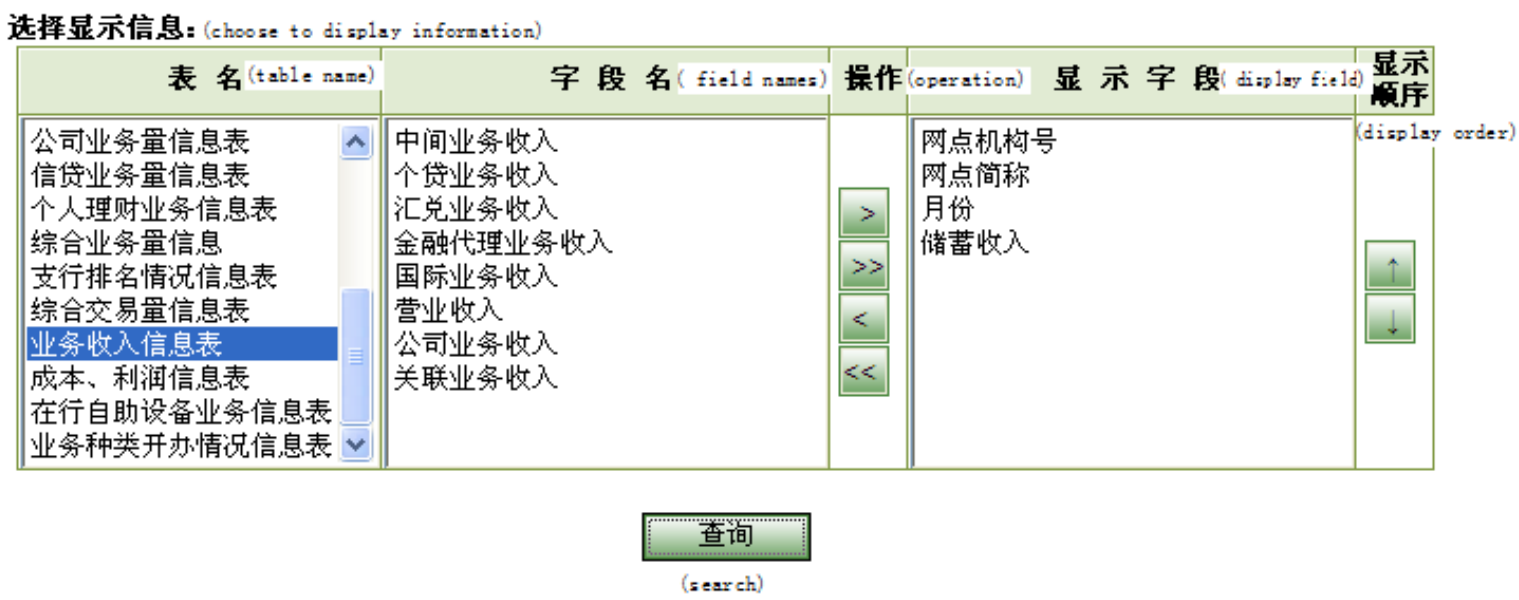

Figure 1 User-defined multi-associated table query conditions and display order selection

The key to achieve user-defined query operation is:

(1)The design of combinational conditional table constructing user-conditional function $\mathrm{F}_{\mathrm{Z}}$. Combinational conditional table contains "Table name"、 "field"、"conditions"、 "input values" four choice boxes. the three choice boxes, "table name"、 "field" 、"conditions" are selected by users. Input value into the box of "input values", then we can completely determinate the comparison components $\left(\mathrm{M}_{\mathrm{i}} \theta \mathrm{V}_{\mathrm{i}}\right)$ in fomula(2).

(2)The design of " $+\left(\right.$ new)" button constructing user-conditional function $\mathrm{F}_{\mathrm{Z}}$. When users press the "new" button, it can add a hyphen $\mathrm{L}_{\mathrm{i}}$ after compare-components $\left(\mathrm{M}_{\mathrm{i}} \theta_{\mathrm{i}} \mathrm{V}_{\mathrm{i}}\right)$. Users then enter the next component $\left(\mathrm{M}_{\mathrm{i}+1} \theta_{\mathrm{i}+1} \mathrm{~V}_{\mathrm{i}+1}\right)$. After users press the "Query" button, it can achieve the user-defined search results according to the user's condition selected in functions $F_{Z}$, then select the options to display, and put its contents displayed on a separate page.

\section{Implementation of user-defined query}

In order to achieve the above algorithm in real projects, we have to first understand the role of the data dictionary and DWR throughout the project.

\subsection{Create a data dictionary for providing selection criteria of multi-table multi-field queries}

The data dictionary is a catalog that users can access records database and application source data. In this paper, the meaning of the data dictionary is a tool used in database design, used to describe the basic design of the table in the database, mainly includes English table names, Chinese table name, English name field, Chinese name field, field data types, field length, primary key, foreign key, if the drop-down menu, drop-down menu name, regular expression and other properties for describing table and contents to be checked. Among them, Chinese table name, Chinese field name is presented to the user to see, really participating in the SQL statement is the English table name、English field name、 the field data types、 field length and drop-down menus when reflecting on the page, they are used to validate the form[3]. If a field is a date type, then the input box will pop up a calendar when you click on to let the users choose; If a field has a drop-down menu, then the input box becomes the focus when using AJAX technology automatically displays the contents of the drop-down menu. 
Drop-down menu name will be associated with a menu table, this table is also a data dictionary table, including English menu name, Chinese menu name and so on.

\subsection{DWR fetch data without refreshing the page from the background}

DWR[4] is a Ajax[5] open-source framework of remote server for improving interaction between web page and Java class, it can allows the code in the browser uses the JAVA function running on the WEB server, just as it is in the browser.

Financial network management information system is based on J2EE architecture, using the open-source SSH2 framework, we write the business logic and database access factory on the WEB server, configure web.xml、struts.xml applicationContext.xml and dwr.xml well, in the browser you don't need to refresh to call JAVA method of server. Meanwhile we can write a lot of js code in the browser, making user-defined query and display of multiple associated table more flexible, more personalized, users can according to their needs choose any query condition, personalize user-defined display items and display order.

Using the above algorithm-based, and merge data dictionary and DWR technology, we finalize user-defined query function.

\section{Conclusion}

After the financial network management information system was successfully developed, through 2 years practical use, it works very well. For researching programming algorithm in the J2EE architecture, there are still many areas need to be explored, we hope to expand further in future research.

\section{References}

[1] QIAO Li, JIANG Huilin, Case search algorithm based on model of user search behavior[J] , Computer Engineering and Applications. 2012,48(6):139-142.

[2] JIA Shi-jie, HUANG Qing-song, MA Shi-xia, Case Retrieval Strategy Based on Grid Clustering[J]. Computer Engineering, 2009,35(10):170-172.

[3] QIAO Li, JIANG Huilin, Case retrieval algorithm based on k-means clustering. Computer Engineering and Applications. 2011,47(4):185-187.

[4] LI Feng, WEI Ying, Case Retrieval Algorithm Based on Preference Information[J]. Computer Engineering, 2008, 34(24): 28-30.

[5] DING Guo-dong, BAI Shuo, WANG Bin, Local Co-occurrence Based Query Expansion for Information Retrieval[J]. Journal Of Chinese Information Processing,2006,20(3):48-53.

[6] YU Chunlei, ZHU Zhengyu, Personalized query expansion method[J]. Computer Engineering and Applications,2012,48(2):119-123.

[7] YANG An-qi, SHAN Zhen-fang, The Design of the Network Management System of Manufacturing Enterprise Based on PB8.0[J]. Light Industry Machinery. 2005(1). 